\title{
On the Bargmann space approach to the extended coupled cluster method for simple anharmonic systems
}

\author{
Document Version \\ Accepted author manuscript
}

Link to publication record in Manchester Research Explorer

\section{Citation for published version (APA):}

Aalto, E., Arponen, JS., \& Bishop, RF. (1990). On the Bargmann space approach to the extended coupled cluster method for simple anharmonic systems. In VC. Aguilera-Navarro (Ed.), Condensed Matter Theories, Vol. 5 (pp. 295-308). Plenum Publishing Corporation.

http://personalpages.manchester.ac.uk/staff/raymond.bishop/RFB_papers/[080] CMT_5(1990)295

\section{Published in:}

Condensed Matter Theories, Vol. 5

\section{Citing this paper}

Please note that where the full-text provided on Manchester Research Explorer is the Author Accepted Manuscript or Proof version this may differ from the final Published version. If citing, it is advised that you check and use the publisher's definitive version.

\section{General rights}

Copyright and moral rights for the publications made accessible in the Research Explorer are retained by the authors and/or other copyright owners and it is a condition of accessing publications that users recognise and abide by the legal requirements associated with these rights.

\section{Takedown policy}

If you believe that this document breaches copyright please refer to the University of Manchester's Takedown Procedures [http://man.ac.uk/04Y6Bo] or contact uml.scholarlycommunications@manchester.ac.uk providing relevant details, so we can investigate your claim.

\section{OPEN ACCESS}




\section{Condensed MATTER THEORIES VOLUME 5}

Edited by

V. C. Aguilera-Navarro

Instituto de Física Teórica-UNESP

São Paulo, Brazil

Plenum Press •New York and London 
$\varphi$ 


\title{
ON THE BARGMANN SPACE APPROACH TO THE EXTENDED
}

\section{COUPLED CLUSTER METHOD FOR SIMPLE ANHARMONIC SYSTEMS}

\author{
E. Aalto*, J.S. Arponen ${ }^{\dagger}$ and R. F. Bishop ${ }^{\#}$ \\ *Physics Computation Unit, University of Helsinki \\ Siltavuorenpenger 20 C, SF-00170 Helsinki, Finland \\ ${ }^{\dagger}$ Department of Theoretical Physics, University of Helsinki \\ Siltavuorenpenger 20 C, SF-00170 Helsinki, Finland \\ \# Department of Mathematics, University of Manchester \\ Institute of Science and Technology, P. O. Box 88 \\ Manchester M60 1QD, England
}

\section{INTRODUCTION}

Bosonic quantum field theories in a Hilbert space can be mapped into classical field theories of complex functions in a particular normed space, the Bargmann Hilbert space. ${ }^{1)}$ Since the theory of complex classical fields is well understood from complex analysis, the approach often affords an intuitively appealing alternative to the more usual canonical or path integral formulations. In the present paper we shall combine this approach with another well-known method of field theory, the coupled cluster method (CCM) of Coester and Kümmel. ${ }^{2)}$ The CCM focuses on the many-body correlations in a quantum system, and introduces a set of linked-cluster amplitudes, $\left\{S_{n}\right\}$, parametrizing the correlated many-body ground state. ${ }^{3)}$

It has been pointed out ${ }^{4)}$ that the CCM equations for the ground state can be obtained from a dynamical variational principle, which can be used to extend the theory into a complete dynamical description of the system, including the CCM average-value functional for arbitrary operators as a central concept. It turned out that in this approach the set of variables, $\left\{\Omega_{n}\right\}$, dynamically conjugate to the amplitudes $\left\{S_{n}\right\}$, are not represented by linked diagrams in the ground state. In another formulation of CCM, the extended CCM (ECCM), ${ }^{4,5)}$ a new set of conjugate variables, $\left\{\sigma_{n}, \tilde{\sigma}_{n}\right\}$, was introduced for the parametrization of the quantum system and shown to be represented by linked diagrams only.

The importance of the linked-cluster properties are naturally related to the problem of size-extensivity and size-consistency (i.e. full separability) of the description 
of the many-body system. ${ }^{6,7)}$ If we consider a local field theory or a many-body system with (sufficiently) short range interactions, the ECCM amplitudes $\left\{\sigma_{n}, \tilde{\sigma}_{n}\right\}$ in the ground state are quasilocal, ${ }^{4,5}$ ) and can be regarded as multilocal coordinates in a classical symplectic complex phase space, the ECCM phase space. The original quantum problem thus becomes mapped into a classical Hamiltonian theory in an appropriate phase space.

Indeed, considering the locality properties of the basic conjugate variables, we may divide the conventional algebraic many-body methods into 3 main categories, in each of which the dynamical equations of motion are obtained from the generic canonical equations

$$
\begin{aligned}
i \frac{d x_{n}}{d t} & =\frac{\partial \bar{H}[x, y]}{\partial y_{n}}, \\
i \frac{d y_{n}}{d t} & =-\frac{\partial \bar{H}[x, y]}{\partial x_{n}} .
\end{aligned}
$$

The canonically conjugate operators $\{x, y\}$ have suitable configuration-space parametrizations in terms of c-number amplitudes $\left\{x_{n}, y_{n}\right\}$, respectively, and the parametrizations of the ket and bra ground states $|\psi\rangle$ and $\langle\vec{\psi}|$ in these cases are

$$
\begin{array}{lll}
\{x, y\}=\{F, \tilde{F}\} ; & |\psi\rangle=F|\phi\rangle ; & \langle\tilde{\psi}|=\langle\phi| \tilde{F} \\
\{x, y\}=\{S, \Omega\} ; & |\psi\rangle=e^{S}|\phi\rangle ; & \langle\tilde{\psi}|=\langle\phi| \Omega e^{-S} \\
\{x, y\}=\{\Sigma, \tilde{\Sigma}\} ; & |\psi\rangle=e^{S}|\phi\rangle ; & \langle\tilde{\psi}|=\langle\phi| e^{S^{\prime \prime}} e^{-S}
\end{array}
$$

where $|\phi\rangle$ is some suitable reference state or cyclic vector. The cases are identified as (I) the configuration interaction (CI) method, (II) the normal CCM (NCCM), and (III) the ECCM. In the last case $\tilde{\Sigma} \equiv S^{\prime \prime}$ and $\Sigma|\phi\rangle=Q e^{S^{\prime \prime}} S|\phi\rangle$, where $Q \equiv I-|\phi\rangle\langle\phi|$. The classical phase space in each of the above cases is in principle equally complicated, but the full locality and separability feature in the ECCM should lead to an effective compactification of the phase space, ${ }^{5 a}$ ) allowing the physically important region to be described in terms of an effective mean field theory of reduced dimensionality.

To learn more about the basic mathematical aspects of the various coupledcluster methods we undertake here a study of two very simple but nontrivial problems: the linear anharmonic oscillator (AO for short) with an $x^{4}$ anharmonicity, and an anharmonic spin system which is introduced as a finite-dimensional model to the anharmonic oscillator with an infinite-dimensional Hilbert space. The anharmonic oscillator is a system for which perturbation theory fails to converge, and the structure of the excitation spectrum changes drastically. ${ }^{8-10)}$ As an interacting field theory the $\mathrm{AO}$ is rather strange and singular because it is not a local field theory; rather, the interaction is maximally non-local. Therefore it is expected to pose an exceptionally hard test for a method such as the ECCM which - with its linked cluster properties - is tailored for systems with normal locality and separability properties.

\section{ANHARMONIC MODEL SYSTEMS}

\section{1. Anharmonic oscillator}

In terms of the canonical creation and annihilation operators the Hamiltonian

$$
H=\frac{1}{2} p^{2}+\frac{1}{2} x^{2}+\frac{\lambda}{4} x^{4}
$$


of the anharmonic oscillator becomes

$$
H=a^{\dagger} a+\frac{1}{2}+\frac{\lambda}{16}\left(a^{\dagger}+a\right)^{4} .
$$

In numerical computation we usually start from the Bogoliubov-transformed optimized form ${ }^{11,12)}$

$$
H=\omega\left[a^{\dagger} a+\frac{\lambda}{16 \omega^{3}}:\left(a^{\dagger}+a\right)^{4}:+\frac{3}{8}+\frac{1}{8 \omega^{2}}\right],
$$

in which $\omega$ is the positive root of equation $\omega^{3}-\omega-\frac{3}{2} \lambda=0$, and the colons denote normal ordering.

In the Bargmann representation we replace $a \rightarrow \frac{d}{d z}, a^{\dagger} \rightarrow z$, and the ket state, such as

$$
|\psi\rangle=F\left(a^{\dagger}\right)|0\rangle
$$

where $|0\rangle$ is the vacuum state defined by $a|0\rangle=0$, will be represented by functions $F(z)$ of the complex variable. ${ }^{13)}$ In the SUB $N$ truncation of the CI method the exact infinite-order holomorphic function $F(z)=\sum_{n=0}^{\infty} F_{n} z^{n}$ is approximated by a polynomial of order $N$. Similarly, in CCM the SUB $N$ approximation to the exact function $S(z)=\sum_{n=1}^{\infty} S_{n} z^{n}$ defined through $F(z)=\exp S(z)$, is a polynomial of order $N$. We observe that the scalar product in the Bargmann representation can be given in any of the following forms, ${ }^{13}$ )

$$
\begin{aligned}
\left\langle 0\left|g(a) f\left(a^{\dagger}\right)\right| 0\right\rangle & =\left.g\left(\frac{d}{d z}\right) f(z)\right|_{z=0} \\
& =\frac{1}{\pi} \int d^{2} z e^{-|z|^{2}} g^{*}(z) f(z) \\
& =\sum_{n} n ! g_{n}^{*} f_{n}
\end{aligned}
$$

Using the Hamiltonian $H\left(a^{\dagger}, a\right) \rightarrow H\left(z, \frac{d}{d z}\right)$ we may write down the energy functional in each of the three methods:

$$
\begin{aligned}
\bar{H}(C I) & =\left.\tilde{F}\left(\frac{d}{d z}\right) H\left(z, \frac{d}{d z}\right) F(z)\right|_{z=0} /\left.\tilde{F}\left(\frac{d}{d z}\right) F(z)\right|_{z=0} ; \\
\bar{H}(C C M) & =\left.\Omega\left(\frac{d}{d z}\right) e^{-S(z)} H\left(z, \frac{d}{d z}\right) e^{S(z)}\right|_{z=0} ; \\
\bar{H}(E C C M) & =\left.e^{S^{\prime \prime}\left(\frac{d}{d z}\right)} e^{-S(z)} H\left(z, \frac{d}{d z}\right) e^{S(z)}\right|_{z=0} \cdot
\end{aligned}
$$

The exact values of the amplitudes $\left(F_{n}, \tilde{F}_{n}, S_{n}, \Omega_{n}, S_{n}^{\prime \prime}\right)$ are found in each case from the condition that $\bar{H}$ is required to be stationary against variations of the respective free variables.

After a lengthy but straightforward calculation the ground-state energy functional in the NCCM and ECCM cases is obtained in the form

$$
\bar{H}=\sum_{n=0}^{M} n ! \Omega_{n} \bar{H}_{n}[S]
$$


where the coefficients are (here $g=\frac{\lambda}{16 \omega^{3}}$ ),

$$
\begin{aligned}
\bar{H}_{n}[S] / \omega= & \delta_{n, 0}\left(\frac{3}{8}+\frac{1}{8 \omega^{2}}\right)+n S_{n} \\
& +g\left\{\delta_{n, 4}+4(n-2) S_{n-2}+6 n(n-1) S_{n}\right. \\
& +4(n+2)(n+1) n S_{n+2}+(n+4)(n+3)(n+2)(n+1) S_{n+4} \\
& +6 \sum_{m} m(n-m) S_{m} S_{n-m} \\
& +12 \sum_{m} m(m-1)(n+2-m) S_{m} S_{n+2-m} \\
& +\sum_{m} m(m-1)(n+4-m)(m+3 n+1) S_{m} S_{n+4-m} \\
& +4 \sum_{m k} m k(n+2-m-k) S_{m} S_{k} S_{n+2-m-k} \\
& +6 \sum_{m k} m k(n+4-m-k)(n+3-m-k) S_{m} S_{k} S_{n+4-m-k} \\
& \left.+\sum_{m k l} m k l(n+4-m-k-l) S_{m} S_{k} S_{l} S_{n+4-m-k-l}\right\} .
\end{aligned}
$$

Equations (2.9)-(2.10) give the energy functional $\vec{H}$ explicitely in terms of the NCCM canonical variables $\left\{S_{n}, \Omega_{n}\right\}$. However, in the ECCM SUB $N$ approximation we do not express $\bar{H}$ explicitely in terms of the canonical variables $\left\{\sigma_{n}, \tilde{\sigma}_{n}\right\}$, but instead use the variables $\left\{S_{n}, S_{n}^{\prime \prime}\right\}$ for convenience. This corresponds to a one-to-one change of variables within the limits of computational accuracy. The ECCM canonical variables $\left\{\sigma_{n}\right\}$ are given by

$$
\sigma_{n}=\sum_{m} \frac{m !}{n !} S_{m} \Omega_{m-n}
$$

where the upper limit of summation is $N$ in a SUB $N$ approximation and infinity in the exact definition.

Above, in equation (2.9) the upper limit of summation is $M=N$ in the NCCM SUB $N$, and $M=4 N-4$ in the ECCM SUB $N$ approximation. In the latter case the amplitudes $\Omega_{n}$ of $\Omega(z) \equiv \exp S^{\prime \prime}(z)$ must be given in terms of the coefficients $\left\{S_{n}^{\prime \prime} \mid n=1, \ldots, N\right\}$. To calculate $\Omega_{n}$ as functions of $\left\{S_{m}^{\prime \prime}\right\}$ and vice versa, we use the recursion formulae

$$
n \Omega_{n}=\sum_{m=1}^{\min (N, n)} m S_{m}^{\prime \prime} \Omega_{n-m}, \quad n=1, \ldots, M .
$$

In practice, for the symmetric AO only the even coefficients are nonzero.

It should be stressed that the CI SUB $N$ approximation simply corresponds to the Sturm-Liouville expansion of the eigenstates of the anharmonic oscillator; i.e. to the diagonalization of $H$ in the subspace spanned by the $N+1$ lowest eigenstates of the linear harmonic oscillator. Therefore, the sequence of CI SUB $N$ results are expected to smoothly converge to the exact limit corresponding to the $\mathrm{AO}$. 


\section{2. Anharmonic spin model}

The Hilbert space of the AO is infinite-dimensional. Therefore the summations in many of the exact untruncated mathematical expressions are infinite, and the question of convergence of such summations naturally arises (see section $\mathrm{V}$ ). We have attempted to gain more understanding on these problems by constructing a finite-dimensional analog to the AO using spin algebra.

We consider the $(2 J+1)$-dimensional spin space corresponding to spin quantum number $J$, and define the operators

$$
\begin{gathered}
j_{0}=J+J_{z}, \\
j_{ \pm}=\frac{1}{\sqrt{2 J}} J_{ \pm}
\end{gathered}
$$

In the limit of large $J$, the commutation relations of $j_{0}, j_{+}$and $j_{-}$are the same as those of $a^{\dagger} a, a^{\dagger}$ and $a$ up to order $\mathcal{O}\left(\frac{\left\langle a^{\dagger} a\right\rangle}{J}\right)$. In the limit $J \rightarrow \infty$ the Hamiltonian

$$
H_{J}=j_{0}+\frac{1}{2}+\frac{\lambda}{16}\left(j_{+}+j_{-}\right)^{4}
$$

should therefore go smoothly into the Hamiltonian (2.2) of the AO, in the sense that any energy eigenvalue $E_{n}$ with fixed $n$ converges to the corresponding $\mathrm{AO}$ energy.

The CI, NCCM and ECCM amplitudes can be defined in the same way as in the $\mathrm{AO}$ case. In particular, the expressions for the amplitudes $\sigma_{n}$ of $\Sigma=\sum_{n} \sigma_{n} j_{+}^{n}$ are now finite sums,

$$
\begin{aligned}
\sigma_{n} & \equiv \frac{1}{K_{n}}\left\langle 0\left|j_{-}^{n} \Omega S\right| 0\right\rangle \\
& =\sum_{m=n}^{2 J} \frac{K_{m}}{K_{n}} S_{m} \Omega_{m-n}
\end{aligned}
$$

where

$$
K_{n}=\frac{n !(2 J) !}{(2 J-n) !(2 J)^{n}} \underset{J \rightarrow \infty}{\rightarrow} n !
$$

\section{NUMERICAL RESULTS}

The NCCM equations for the anharmonic oscillator have been previously solved numerically, ${ }^{11,12,14)}$ but no ECCM solutions have been published so far. In order to compare the convergence and accuracy, we solved numerically the $\mathrm{AO}$ using all three methods. The ECCM and NCCM equations were solved by Newton's iteration, and the CI ground state by inverse iteration. We used VAX FORTRAN with quadruple precision for the ground state, and double precision NAG routines for the excitation energies (F02AFF for NCCM and ECCM, and F02AAF for CI). In each case the excited-state energies were calculated by using the dynamical matrix obtained from the second-order derivatives of the energy functional, as explained in an accompanying paper in this volume. ${ }^{15)}$

In Fig. 1 we show the results for the first six eigenvalues corresponding to evenparity eigenstates at $\lambda=0.05$. The accuracy is indicated by $-\lg \left|1-\frac{E}{E_{\text {exact }}}\right|$ plotted as function of the truncation index $N$. Thus the ordinate represents directly the 

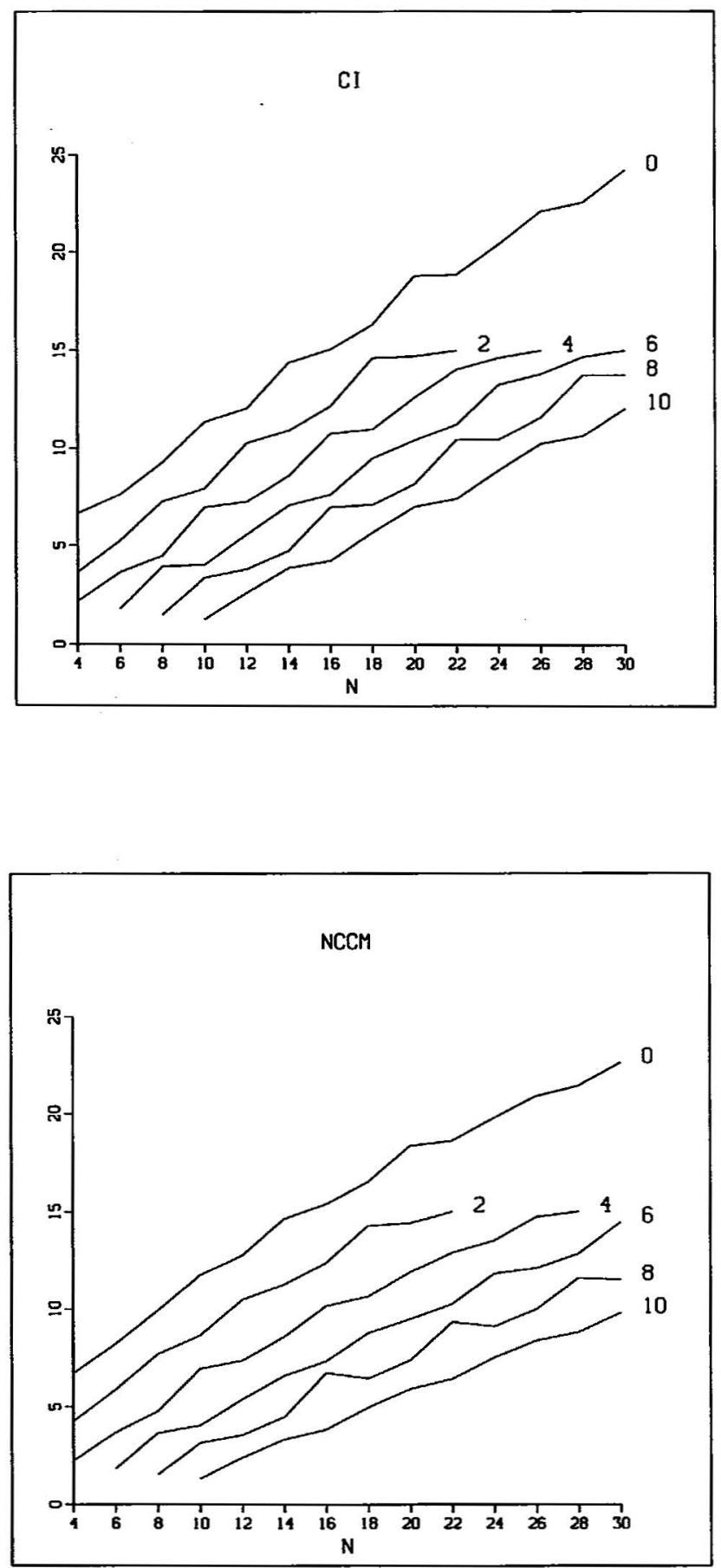

Fig. 1. Accuracy of the energies of the six lowest even eigenstates of the anharmonic oscillator with $\lambda=0.05$ as functions of truncation index $N$. The excited-state results are given up to the point where full double-precision accuracy is reached. 


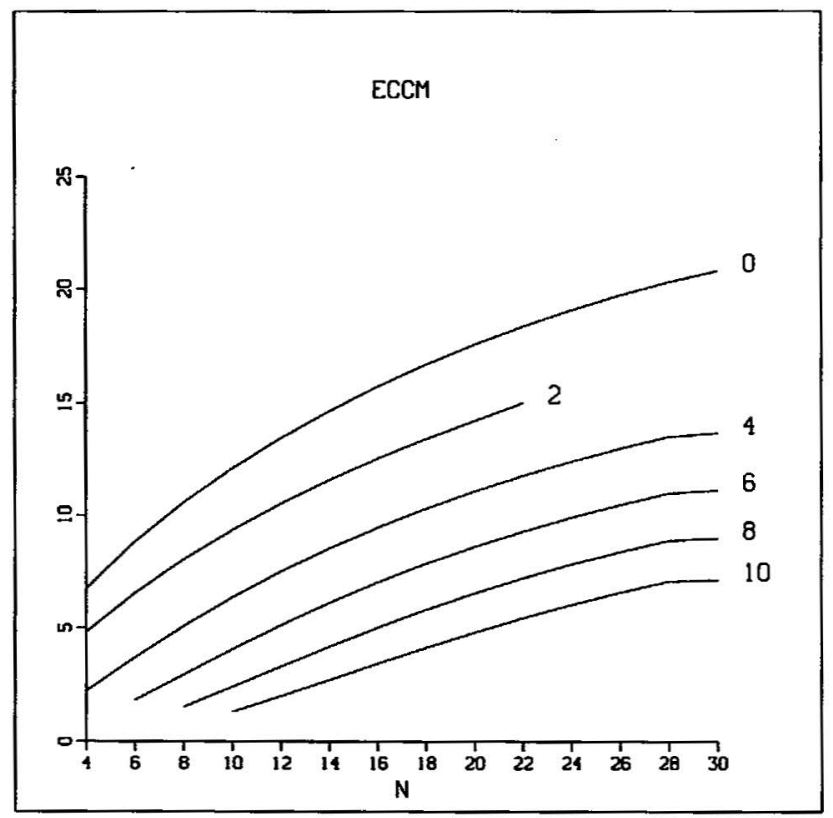

Fig. 1. Continued.

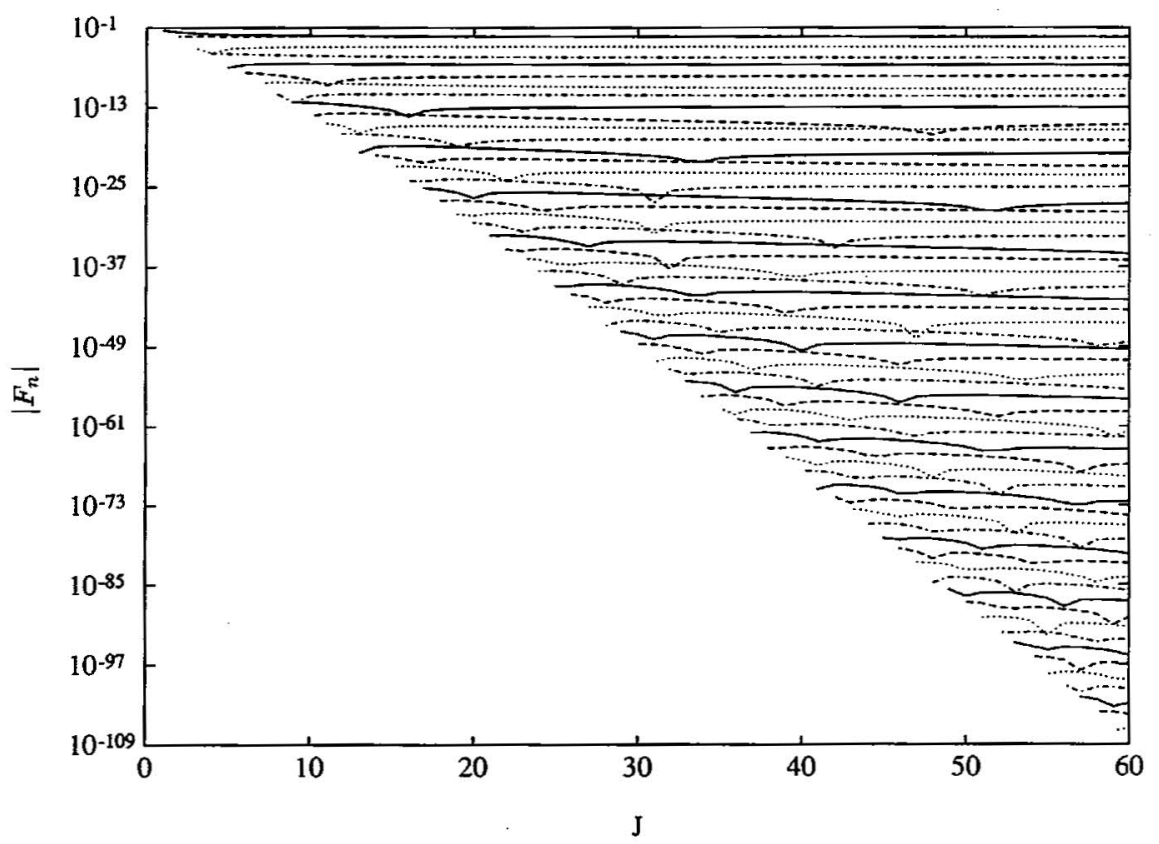

Fig. 2. Amplitudes for the anharmonic spin model as functions of $J$ at $\lambda=1$. Only even-indexed amplitudes up to $n \leq N=2 J$ exist. 

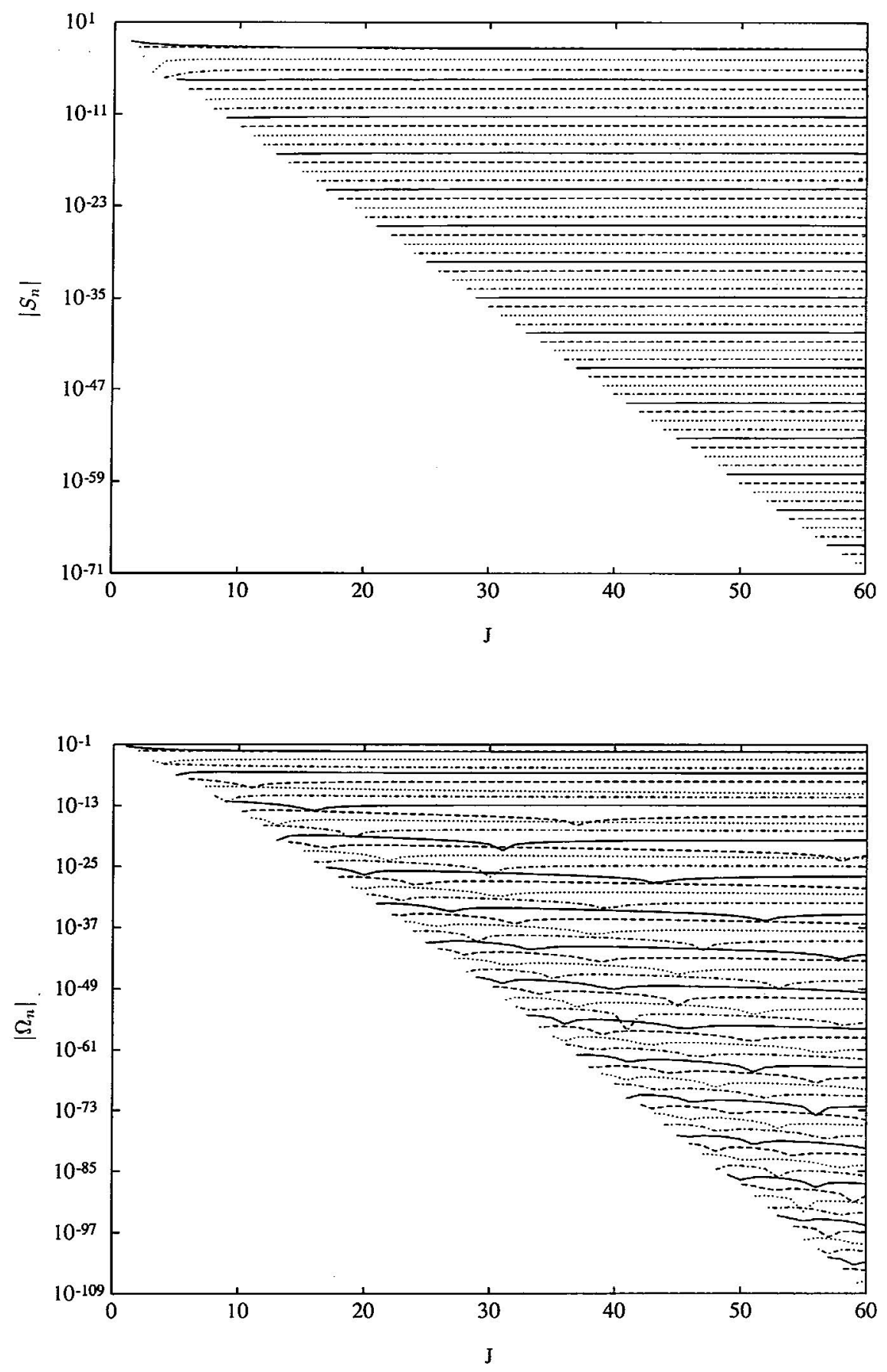

Fig. 2. Continued. 

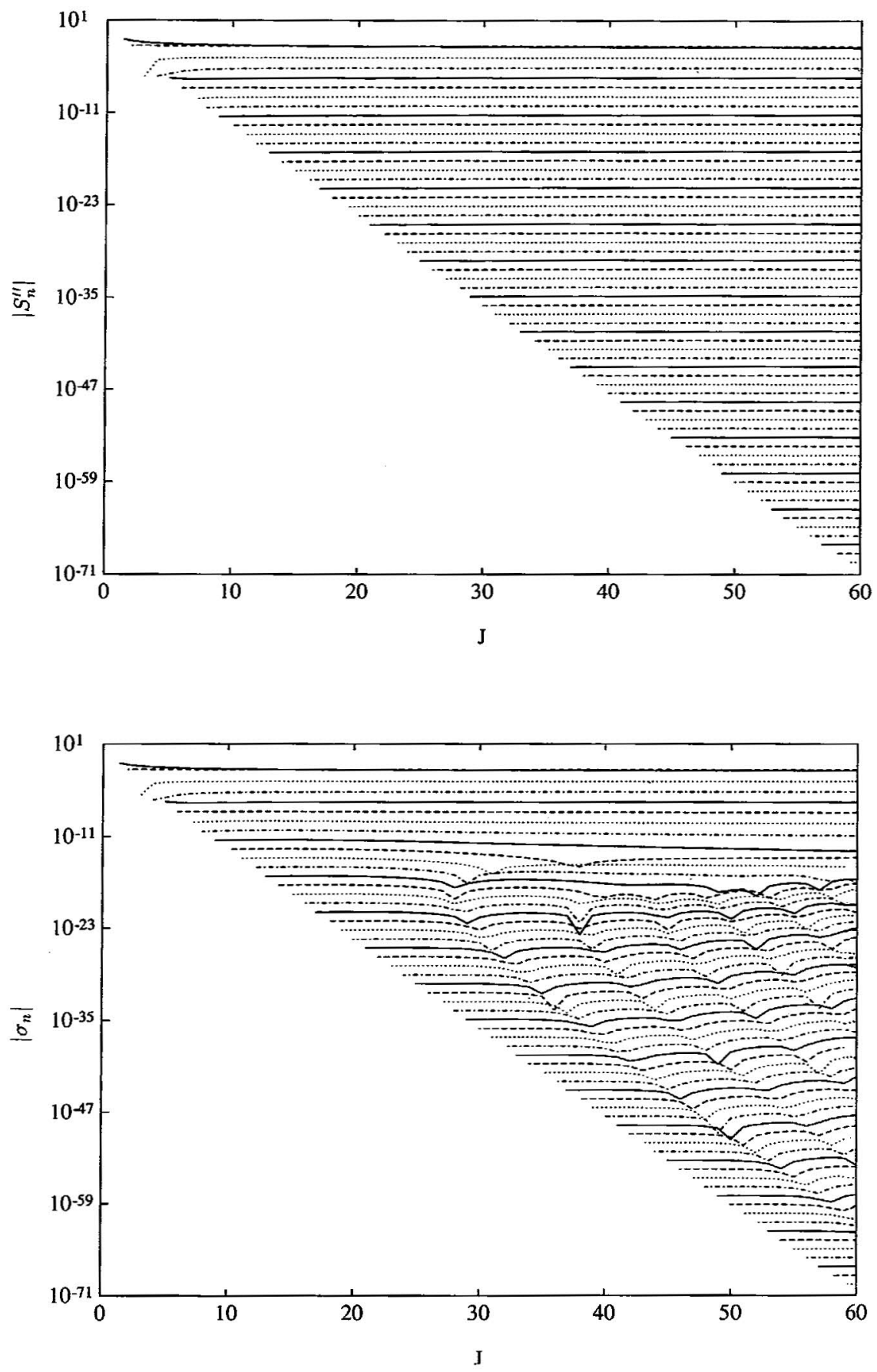

Fig. 2. Continued. 
number of correct significant digits. It can be seen that for a small $\lambda$ ECCM is competitive in accuracy with the other methods, and the increase of accuracy with increasing $N$ is remarkably smooth. For larger $\lambda$ ECCM gives results clearly inferior to the other methods, and we did not succeed to find convergence for $N$ larger than 12 or 14.

We have not yet been able to establish whether these problems are caused by a real disappearence of the solution or by some instability of the computation. One possible cause of instability is the recursion formula (2.12). We have found out numerically, and in section IV shall demonstrate analytically that the calculation of $\left\{S_{n}^{\prime \prime}\right\}$ from $\left\{\Omega_{n}\right\}$ is essentially irreversible. The reverse calculation is needed in the ECCM equations, which could be one of the causes of the problems. It can be stated, however, that the reasons must be more complicated than just due to accumulation of rounding errors, since a calculation using only the G_FLOATING double precision did not give essentially worse results. We also examined the disappearence of the solution by letting $\lambda$ increase with constant $N$ and using the results from the previous step as input for the next. We found out that the disappearence was preceded by the largest exitation energy becoming absurdly large and the two next largest coalescing and becoming complex conjugates signifying a dynamical instability. The ground state energy from the ECCM always seemed to be an upper limit, unlike in the case of the NCCM. ${ }^{11)}$ Also, the excited-state energies of the stable ECCM solution were found to be upper limits to the exact ones, whereas the excited NCCM energies did not have this property. This explains partly the smooth improvement of the ECCM results seen in Fig. 1. Finally, we note that we found no evidence for the non-uniqueness of solutions, as in the NCCM ${ }^{11,14)}$; we either found one solution or none.

The anharmonic spin system is solvable by ordinary matrix methods, like the ones used for the $A O$ in the CI case, and it therefore allows us to find the exact values of all the CI, NCCM and ECCM amplitudes. In Fig. 2 we plot typical results for all the amplitudes $F_{n}, \Omega_{n}, S_{n}, S_{n}^{\prime \prime} \equiv \tilde{\sigma}_{n}$, and $\sigma_{n}$ for a range of dimensions J up to 60 , for a fixed interaction strength $\lambda=1$ (the results for all $\lambda$ were qualitatively similar).

It is found out that the amplitudes $F_{n}$ and $\Omega_{n}$ always behave qualitatively in a fairly identical way. In particular, their convergence to the limits at $J \rightarrow \infty$ is often very slow as the dimension $J$ increases. In contrast, the convergence of the coupledcluster amplitudes $S_{n}$ and $S_{n}^{\prime \prime}$ is very quick. This shows that these amplitudes provide a very stable and characteristic set of parameters for the description of the ground state. The stability of the calculation of $S_{n}^{\prime \prime}$ gives us also a clue concerning the instability of the reverse calculation, which could explain the problems mentioned above. In fact, as will be proven in the next section, the values of $S_{n}^{\prime \prime}$ for large $n$ decrease only as a geometric series, whereas the amplitudes $\Omega_{n}$ decrease much faster. In the limit of large $n$ the exact amplitudes $\Omega_{n}$ become negligible in comparison with $S_{n}^{\prime \prime}$ and the recursion formula (2.12) cannot be used to calculate $\Omega_{n}$ from the set $\left\{S_{n}^{\prime \prime}\right\}$. The stabilized values of $F_{n}, \Omega_{n}, S_{n}$ and $S_{n}^{\prime \prime}$ in the limit $J \rightarrow \infty$ obviously provide also the exact solution of the AO problem. The exact $S_{n}$ amplitudes for the AO problem have been evaluated and compared with their NCCM approximations in Ref. 12.

The amplitudes $\sigma_{n}$ are found to behave in a different way. Their values do not seem to stabilize as $J \rightarrow \infty$, up to the limit which was possible to reach with our numerical accuracy. Also, it was found that the higher terms in the sum (2.15) yield significant contributions to $\sigma_{n}$ at high $J$. Thus it seems that the exact $\sigma_{n}$-amplitudes of the AO case cannot be calculated from this particular finite-dimensional model in its infinite-dimensional limit. 


\section{ANALYTIC CONSIDERATIONS}

The holomorphic state function $F(z)$ is known, on general grounds, to be an entire function of order $\leq 2$. Specifically, in our case it has the precise expression ${ }^{8,13}$ )

$$
F(z)=\pi^{-\frac{1}{4}} e^{-\frac{1}{2} z^{2}} \int_{-\infty}^{\infty} d x e^{\sqrt{2} z x-\frac{1}{2} x^{2}} \psi(x)
$$

where $\psi(x)$ is the suitably normalized (i.e. $\langle 0 \mid \psi\rangle=1$ ) coordinate-space wave function. It is proven elsewhere ${ }^{13)}$ that the function $F(z)$ corresponding to the ground state of the AO must have infinitely many distinct zeroes $\left\{z_{m}\right\}$ which, asymptotically far from the origin, are located at the points $\pm i y_{m}$ where

$$
y_{m} \underset{m \rightarrow \infty}{\rightarrow}\left(\frac{\lambda}{2}\right)^{\frac{1}{6}}\left(\frac{3 \pi m}{2}\right)^{\frac{2}{3}} .
$$

The function $e^{\frac{1}{2} z^{2}} F(z)$ is an entire function of order $\frac{3}{2}$ and, on using its Hadamard decomposition, it is straightforward to show that the exact CCM amplitude $S(z)$ of the $\mathrm{AO}$ must be of the form

$$
S(z)=-\frac{1}{2} z^{2}+\sum_{m}\left[\ln \left(1-\frac{z}{z_{m}}\right)+\frac{z}{z_{m}}\right] .
$$

It is now evident that the state $S\left(a^{\dagger}\right)|0\rangle$ is not normalizable, and therefore the CCM operator $S\left(a^{\ddagger}\right)$ cannot be qualitatively considered as a 'small' correlation operator.. Also, the coefficients $S_{n}$ for high $n$ are determined by the zero closest to the origin. The sequence $n S_{n}$ must, indeed, be asymptotically a geometric series such that $\lim _{n \rightarrow \infty} \sqrt[n]{n\left|S_{n}\right|}=\rho^{-1}$, where $\rho$ is the distance of the nearest zero.

The function $\Omega(z)$ can be given in terms of the wave function $\psi(x)$ as the integral ${ }^{13}$ )

$$
\Omega(z)=N^{-2} e^{-\frac{z^{2}}{4}} \int_{-\infty}^{\infty} d x e^{\frac{x z}{\sqrt{2}}} \psi^{*}(x) \psi\left(x-\frac{z}{\sqrt{2}}\right),
$$

where $N^{2} \equiv \int d x|\psi(x)|^{2}$. For the ground state of the $\mathrm{AO}$ this function is also an entire function.

We now calculate the coefficients $\sigma_{n}$ of the ground-state amplitude $\Sigma(z)=$ $\sum_{n} \sigma_{n} z^{n}$ from the definition (cf. section I and equation 2.11)

$$
\sigma_{n}=\left.\frac{1}{n !} \frac{d^{n}}{d z^{n}} \Omega\left(\frac{d}{d z}\right) S(z)\right|_{z=0} \quad n \geq 1
$$

Using Eq. (4.3) for $S(z)$ one can proceed either by a Fourier integral or by a Borel summation method. Whichever method is chosen, the calculations are rather involved, and the details are given elsewhere. ${ }^{13}$ ) We first define an odd function $R(x)$ by

$$
R(x)=\delta^{\prime}(x)-\frac{1}{2} \operatorname{sgn}(x) \sum_{m} e^{-x z_{m}}
$$

for $x \in \mathbb{R}$. Here $\delta(x)$ is the Dirac delta, and $R(x)$ is obviously a distribution. The infinite sum over the zeroes of $F(z)$ is formally divergent and must be properly interpreted. Indeed, the sum must first be partitioned into terms which are analytic 
in either the upper or the lower $x$-halfplanes. The boundary values of these analytic functions then enter in $R(x)$. Using this definition we find the NCCM and ECCM amplitudes in the form

$$
\begin{aligned}
& S^{\prime}(z)=\int_{-\infty}^{\infty} d x R(x) \sinh (x z) \\
& \Sigma^{\prime}(z)=\int_{-\infty}^{\infty} d x \rho(x) \sinh (x z)
\end{aligned}
$$

where $\rho(x)=R(x) \Omega(x)$. The amplitudes $S_{n}$ and $\sigma_{n}$ are thus given by the moments of the distributions $R(x)$ and $\rho(x)$, respectively.

Using the above representations it is possible to formulate a generating function for the expectation values of arbitrary normal-ordered operators. We define

$$
\begin{aligned}
A(u, v) & \equiv\left\langle e^{u a^{\dagger}} e^{v a}\right\rangle \\
& =\left\langle 0\left|e^{S^{\prime \prime}(a)} e^{-S\left(a^{\dagger}\right)} e^{u a^{\dagger}} e^{v a} e^{S\left(a^{\dagger}\right)}\right| 0\right\rangle .
\end{aligned}
$$

Using this function the average value of e.g. the normal-ordered Hamiltonian $H\left(a^{\dagger}, a\right)$ is

$$
\bar{H}=\left.H\left(\frac{\partial}{\partial u}, \frac{\partial}{\partial v}\right) A(u, v)\right|_{u=v=0} .
$$

.The results derived are

$$
A(u, v)=\sum_{n=0}^{\infty} \frac{1}{n !} \prod_{i=1}^{n}\left[\int_{-\infty}^{\infty} d x_{i} R\left(x_{i}\right) \frac{e^{v x_{i}}-1}{x_{i}}\right] \Omega\left(u+x_{1}+\cdots+x_{n}\right)
$$

for NCCM and

$$
\begin{aligned}
A(u, v)= & \sum_{n=0}^{\infty} \frac{1}{n !} \prod_{i=1}^{n}\left[\int_{-\infty}^{\infty} d x_{i} \rho\left(x_{i}\right) \frac{e^{v x_{i}}-1}{x_{i}}\right] \\
& \quad \times \exp \left[\tilde{\Sigma}\left(u+x_{1}+\cdots+x_{n}\right)-\tilde{\Sigma}\left(x_{1}\right)-\cdots-\tilde{\Sigma}\left(x_{n}\right)\right]
\end{aligned}
$$

for ECCM. In each case the function gives expressions in closed form for the averages in terms of the pertinent free variables; the amplitudes $S_{n}$ or $\sigma_{n}$ appear through the moments of the functions $R(x)$ or $\rho(x)$, respectively. When formally expanded, these expressions represent the particular linking and connectivity properties of the corresponding NCCM or ECCM diagrams and their vertices. Precise parallels can clearly be drawn with the well known linked (L) or double-linked (DL) expansions for an average value of an arbitrary operator $\mathcal{O}$, given in the operator forms ${ }^{3-5}$ )

$$
\begin{aligned}
\overline{\mathcal{O}} & =\sum_{n=0}^{\infty} \frac{1}{n !}\left\langle 0\left|\Omega\left\{\mathcal{O} S^{n}\right\}_{L}\right| 0\right\rangle \\
& =\sum_{n=0}^{\infty} \frac{1}{n !}\left\langle 0\left|e^{\bar{\Sigma}}\left\{\mathcal{O} \Sigma^{n}\right\}_{L}\right| 0\right\rangle_{D L}
\end{aligned}
$$

for the NCCM and ECCM, respectively. 
It is evident that the SUB $N$ truncations in the coupled cluster methods are potentially hazardous because the asymptotic analytic forms of the functions $e^{ \pm S}$ or $e^{S^{\prime \prime}}$ become severely distorted from their exact behaviour. E.g., the states $e^{S}|0\rangle$ are not normalizable in the SUB $N$ approximations with $N \geq 3$. Thus the SUB $N$ approximations imply excursions into more general linear spaces outside the Hilbert space of normalizable functions. For this purpose the Bargmann representation in terms of functions of complex variable is very well suited. The exact function $F=e^{S}$, which in our case is an entire function of order 2, may nevertheless be very well represented by an approximate SUB $N$ function $\exp S^{(N)}$, where $S^{(N)}$ is a polynomial of degree $N$, in a part of the complex plane around the origin, although far away the asymptotic behaviour is seriously wrong. For instance, the NCCM method focuses attention on the behaviour of the function $e^{-S} H e^{S}$ just around the origin, requiring it to be constant up to the derivatives of order $N$ at $z=0$. This feature allows us to understand the practical success of the method in spite of the incorrect asymptotic behaviour. The normalizability problem of the NCCM SUB $N$ approximation has been discussed also in Refs. 11-12. In the case of ECCM, the stationarity condition is slightly more complicated, and obviously more sensitive to the asymptotic behaviour, making the SUB $N$ truncations even more risky.

The exact amplitudes $S_{n}$ and $S_{n}^{\prime \prime}$ for the AO problem can be easily computed either from the anharmonic spin analog or from the CI solution of the AO problem itself. However, it is doubtful whether they can be found from the SUB $N$ solutions in the limit $N \rightarrow \infty$ (see also Ref. 12). This fact is rather significant and indicates that the sequence of SUB $N$ truncations may not ultimately converge for either NCCM or ECCM. We interprete this to emphasize that 'particle number', or the number of correlated fields in a 'multilocal' amplitude, is not a good concept in a field theory with no particle number conservation. So far we have not tried to evaluate the exact ECGM amplitudes $\sigma_{n}$. They cannot be obtained (at least to our numerical accuracy) from the finite-dimensional spin analog. Nevertheless, they can be computed from the exact analytic expressions given above. This would obviously be a very challenging numerical enterprise.

On the basis of our numerical evaluations, the various coupled-cluster methods at low and intermediate truncation indices $N$ up to SUB $6 \ldots 12$ provide a very good approximation for the energies of the ground state and the lowest excited states for all values of the coupling constant $\lambda$. In particular, for small values of the coupling constant the accuracy typically increases in the order $\mathrm{CI} \rightarrow \mathrm{NCCM} \rightarrow \mathrm{ECCM}$, whereas the order is opposite for large coupling constants. However, for high SUB $N(N \rightarrow \infty)$ the accuracy continues to improve indefinitely only in CI, whereas the NCCM stagnates to lower accuracy. In the ECCM the accuracy ceases to improve at an even lower truncation level (for small $\lambda$ ), or the solution is not even found for $N \gtrsim 14$ (for higher $\lambda$ ). Although the numerical difficulties in the limit $N \rightarrow \infty$ prevent us from very decisive conclusions, it seems obvious that the SUB $N$ approximations are completely safe and convergent only in the CI case. At fixed $\lambda$, therefore, the convergence with respect to the limit $N \rightarrow \infty$ improves in the order ECCM $\rightarrow$ NCCM $\rightarrow$ CI.

Although the SUB $N$ approximations are analytically somewhat questionable for the coupled-cluster methods, we have been able to show that the NCCM and ECCM methods perform rather satisfactorily even in the difficult case of the anharmonic oscillator, which has no attributes of locality, separability, or size-extensivity. We have not been able to demonstrate that the SUB $N$ sequence of approximations ultimately converges for NCCM and ECCM; on the contrary, we were unable to find convergent 
solutions to ECCM for large $N$ in the case of higher $\lambda$. Nevertheless, the ECCM results for all the eigenenergies $E_{n}$ were, quite remarkably, found to be upper limits to the exact values. This is by no means an expected result, because the ECCM (as well as NCCM) is manifestly a non-Hermitian formulation of the many-body problem.

\section{REFERENCES}

1) V. Bargmann, Comm. Pure Appl. Math. 14: 180, 187 (1961); ibid. 20: 1 (1967); Rep. Math. Phys. 2: 221 (1971).

2) F. Coester, Nucl. Phys. 7: 421 (1958); F. Coester and H. G. Kümmel, Nucl. Phys. 17: 477 (1960).

3) For reviews see: R. F. Bishop and H. G. Kümmel, Phys. Today $\underline{40}$ (No. 3): 52 (1987); H. G. Kümmel in "Nucleon-Nucleon Interaction and Nuclear Many-Body Problems", S. S. Wu and T. T. S. Kuo (eds.), World Scientific, Singapore (1984), p. 46; H. G. Kümmel, K. H. Lührmann and J. G. Zabolitzky, Phys. Rep. 36C: 1 (1978). See also references 4,5 .

4) J. Arponen, Ann. Phys. (NY) 151: 311 (1983).

5) J. S. Arponen, R. F. Bishop, and E. Pajanne, Phys. Rev. A36: 2519 (1987); ibid. 2539 (1987); J. Arponen, R. F. Bishop and E. Pajanne in "Condensed Matter Theories", Vol. 2, P. Vashishta, R. K. Kalia and R. F. Bishop (eds.), Plenum, New York (1987), p. 357.

6) H. Primas, in "Modern Quantum Chemistry", Vol. II, O. Sinanoglu (ed.), Academic, New York (1965), p. 45; R. J. Bartlett and G. D. Purvis, Phys. Scr. 21: 255 (1980).

7) I. Lindgren and D. Mukherjee, Phys. Rep. 151: 93 (1987); R. Chowdhuri, D. Mukherjee and M. D. Prasad, in "Aspects of Many-Body Effects in Molecules and Extended Systems", D. Mukherjee (ed.), Springer, Berlin (1989), p. 3.

8) C. M. Bender and T. T. S. Wu, Phys. Rev. 184: 1231 (1969); Phys. Rev. D7: 1620 (1973); Phys. Rev. Letters 27: 461 (1971).

9) B. Simon, Ann. Phys. (NY) 묘: 76 (1970).

10) F. T. Hioe, D. MacMillen and E. W. Montroll, Phys. Rep. 43: 305 (1978); R. Balian, G. Parisi and A. Voros, Phys. Rev. Letters 41: 1141 (1978); S. K. Bose and D. N. Tripathi, Fortschr. Phys. 31: 131 (1983).

11) U. B. Kaulfuss and M. Altenbokum, Phys. Rev. D33: 3658 (1986).

12) H. G. Kümmel in "Condensed Matter Theories", Vol. 3, J. S. Arponen, R. F. Bishop and M. Manninen (eds.), Plenum, New York (1988), p. 21.

13) J. S. Arponen and R. F. Bishop, to be published.

14) R. F. Bishop and M. F. Flynn, Phys. Rev. A38: 2211 (1988); R. F. Bishop, M. C. Boscá and M. F. Flynn, Phys. Lett. A132: 440 (1988).

15) R. F. Bishop, N. I. Robinson, and J. Arponen, this volume. 